This is an electronic reprint of the original article. This reprint may differ from the original in pagination and typographic detail.

Author(s): von Bonsdorff, Mikaela; Rantanen, Taina; Laukkanen, Pia; Suutama, Timo; Heikkinen, Eino

Title: $\quad$ Mobility limitations and cognitive deficits as predictors of institutionalization among community-dwelling older people.

Year: $\quad 2006$

Version:

Please cite the original version:

von Bonsdorff, M., Rantanen, T., Laukkanen, P., Suutama, T., \& Heikkinen, E. (2006). Mobility limitations and cognitive deficits as predictors of institutionalization among community-dwelling older people.. Gerontology, 52, 359-365.

https://doi.org/10.1159/000094985

All material supplied via JYX is protected by copyright and other intellectual property rights, and duplication or sale of all or part of any of the repository collections is not permitted, except that material may be duplicated by you for your research use or educational purposes in electronic or print form. You must obtain permission for any other use. Electronic or print copies may not be offered, whether for sale or otherwise to anyone who is not an authorised user. 


\section{Mobility Limitations and Cognitive Deficits as Predictors of Institutionalization among Community-Dwelling Older People}

Mikaela von Bonsdorff, $\mathrm{MSc}^{1}$, Taina Rantanen $\mathrm{PhD}^{1}$, Pia Laukkanen MD, $\mathrm{PhD}^{2}$, Timo Suutama $\mathrm{PhD}^{3}$, Eino Heikkinen $\mathrm{MD}, \mathrm{PhD}^{1}$

${ }^{1}$ Finnish Centre for Interdisciplinary Gerontology, Department of Health Sciences, University of Jyväskylä, Finland

${ }^{2}$ Sotainvalidien Sairaskoti (Health care and rehabilitation center for war veterans), Finland

${ }^{3}$ Department of Psychology, University of Jyväskylä, Finland

\section{Corresponding author:}

Mikaela von Bonsdorff

Finnish Centre for Interdisciplinary Gerontology

Department of Health Sciences

University of Jyväskylä

P.O. Box 35 (Viveca)

FIN-40014 Jyväskylä University

Tel. +358 142604596

Fax +358 142604600

E-mail mikaela.bonsdorff@sport.jyu.fi

Short title: Co-occurring limitations and risk for institutionalization

Key words: mobility limitations, cognitive deficits, institutionalization, older people 


\title{
Mobility Limitations and Cognitive Deficits as Predictors of Institutionalization among Community-Dwelling Older People
}

\begin{abstract}
Purpose. Mobility limitations and cognitive disorders have often been observed as risks for institutionalization. However, their combined effects on risk of institutionalization among initially community-dwelling older people have been less reported.
\end{abstract}

Design. A prospective cohort study with 10-year surveillance on institutionalization.

Subjects. Study population $(n=476)$ consisted of 75 - and 80 -year-old people who were community-dwelling, had not been diagnosed with dementia, and participated in tests on walking speed and cognitive capacity at a research centre.

Measures. Cognitive capacity was measured with three validated psychometric tests that were from the Wechsler Adult Intelligence Scale, Wechsler Memory Scale and Schaie-Thurstone Adult Mental Abilities Test. Mobility was measured with walking speed over a 10 meter distance. Exclusive distribution based study groups were formed with cut-offs at the lowest third as follows: no limitation, solely mobility limitation, solely cognitive deficits, and combined mobility limitation and cognitive deficits. Cox proportional hazards model was used to determine the relative risks of institutionalization for the study groups. 
Results. Eleven percent of the participants were institutionalized during the 10-year surveillance. The risk for institutionalization was 4.9 times greater (95\% confidence interval 2.1-11.2) for those who had co-existing mobility limitations and cognitive deficits than for those with no limitations.

Conclusions. The findings show that the accumulation of limitations in physical and cognitive performance substantially decrease the possibility for a person remaining at home. This might be due to a decreased reserve capacity and ineffective compensatory strategies. Therefore, interventions targeted to improve even one limitation or prevent accumulation of these risk factors could significantly reduce the risk of institutionalization. 


\section{INTRODUCTION}

Previous longitudinal research has identified several predictors of institutionalization for community-dwelling older persons. Some of the most frequently reported predictors are a greater age, [1] lower cognitive function and dementia [2-4], lower ADL or physical functioning [4-6], female gender [7], not being married or living alone [8], absence of informal or formal caregiver [9-10] and a higher number of illnesses and injuries [1,11].

The theoretical model of the disablement process [12] suggests that impairments lead via functional limitations to subsequent disability, which adds to the risk of institutionalization [13]. Mobility limitations increase the risk of institutionalization and other adverse outcomes for older persons [14-15]. In addition, cognitive disorders, such as mild cognitive impairment and dementia, have been shown to be strong predictors of institutionalization among older persons $[2,6]$. Moreover, older people often tend to suffer from multiple impairments and limitations simultaneously, all of which increase the risk of institutional care. However, the combined effects of co-occurring impairments and limitations on institutionalization have not been studied to our knowledge. This prospective study examined the combined effects of mobility and cognitive capacity on the risk of institutionalization among initially community-dwelling 75- and 80-year-old people over a 10-year surveillance period. 


\section{METHODS}

The data come from a prospective cohort study called the Evergreen project described in detail elsewhere [16]. Target population, 617 persons, consisted of two whole birth-year cohorts (1914 and 1910) who were at baseline 75- and 80-year-old residents of the City of Jyväskylä, Finland. The current study population consisted of those 476 community-dwelling residents who participated in tests at the research centre and did not have a diagnosis of dementia. The 203 persons not included in the study population were either already institutionalized $(n=21)$, did not come to the research centre $(n=180)$, or had a diagnosis of dementia $(n=2)$. They belonged more frequently to the older age cohort (50\% vs. $40 \%, \mathrm{p}=0.031$ ) and had more long-term diseases (3.2, SD 1.5 vs. 2.5 , SD $1.6, \mathrm{p}=0.003)$ compared to the study population.

The outcome of this study was institutionalization. The date of institutionalization was the date when care in a hospital or nursing home staffed around the clock had exceeded 90 days and the subject was in need of continuous care. Dates of permanent institutionalization decisions were collected from local registers. Non-institutionalized participants were included in the analysis until the date of their death or the end of the surveillance period, whichever occurred first. Dates on deaths that occurred during the surveillance period were obtained from the national Population Register Centre. Recordings of institutionalizations and deaths covered the period between January 1, 1989, and December 31, 2000. The study was granted permission from the Ethical committee of Central Finland Health Care District. Each participant signed an informed consent.

At baseline, mobility limitation was assessed using a maximal walking speed test over a 10 meter distance timed with a stop-watch [17]. Participants were allowed 2-3 meters acceleration before 
the start-line. They were encouraged to walk as fast as possible without risking their health and were allowed to use normal walking aids when necessary.

Cognitive capacity was evaluated with a battery of psychometric tests including 1) Digit Span from Wechsler Memory Scale [18] for testing short-term memory, 2) Digit Symbol from Wechsler Adult Intelligence Scale (WAIS) [19] for testing psychomotor speed affected by visual perception and learning, and 3) a modified oral version of Word Fluency from Schaie-Thurstone Adult Mental Abilities Test [20] for testing verbal fluency and flexibility. Psychometric tests were performed at the research centre by trained psychologists. For statistical analysis, a principal component was formed from the above mentioned three tests indicating cognitive capacity. The first principal component explained $65.4 \%$ of the total variance and was selected for the analysis.

Age- and gender-specific distribution-based cut-off values were used to categorize participants into groups with impaired or normal function. The lowest third of each distribution was classified as having mobility limitations or cognitive deficits. Cut-offs for maximal walking speed for 75-year-old men and women were 1.59 and $1.26 \mathrm{~m} / \mathrm{s}$ and for 80 -year-old men and women 1.38 and $1.19 \mathrm{~m} / \mathrm{s}$, respectively. Our cut-offs are in line with those of Shinkai et al. [21] who divided their community-dwelling study participants into quartiles with $1.34 \mathrm{~m} / \mathrm{s}$ and 1.08 $\mathrm{m} / \mathrm{s}$ as cut-offs for the lowest quartile for over 75-year-old men and women, respectively. The cognitive capacity principal component was dichotomized into the lowest third vs. middle and highest thirds. Accordingly, four exclusive study groups were formed indicating 1) no limitation, 2) mobility limitation, 3) cognitive deficits, and 4) mobility limitation and cognitive deficits.

Potential confounders selected based on previous research findings included full-time education (6 years or less vs. more than 6 years) and living alone (yes/no). Comorbidity was indicated by 
the number of long-term diseases at baseline. Information was collected in face-to-face interviews conducted at participants' homes. Participants underwent a half-hour physician consultation at the research centre were self-reported long-term diseases and medication were confirmed and a physical examination was performed. In the comorbidity index, the sum of all diagnoses of long-term conditions which had lasted over three months was calculated and dichotomized for the analysis ( 2 or less vs. 3 or more).

\section{STATISTICAL ANALYSIS}

Comparisons between those who were institutionalized, who survived without being institutionalized and those who died without first being institutionalized were performed with cross-tabulation $\chi^{2}$-testing for dichotomous variables and with one-way analysis of variance for continuous variables. The institutionalization rates were calculated by dividing the number of institutionalizations by the number of person-years of follow-up. Relative risk of institutionalization was estimated using Cox proportional hazards models. Risk ratios and $95 \%$ confidence intervals were estimated for each variable that was entered into the model with $\mathrm{p}<.10$. 


\section{RESULTS}

The mean duration of the follow-up was 2728 days with a range of 58 to 4017 days. At the end of the 10-year surveillance period, of the 476 participants who were community-dwelling at baseline, 52 persons had been institutionalized, 209 remained community-dwelling and 215 had died without prior institutionalization.

Demographic and health characteristics of those who during the surveillance period remained community-dwelling, were institutionalized or died without institutionalization first are presented in Table 1. Almost $80 \%$ of the institutionalized persons were women, while the corresponding number was $70 \%$ for those who remained community-dwelling and $60 \%$ for those who died without prior institutionalization. At baseline, about $60 \%$ of those who stayed community-dwelling throughout the surveillance had three or more long-term diseases whereas the corresponding number was about $70 \%$ for those who were institutionalized and more than 80 $\%$ for those who died. Those who remained community-dwelling had the highest average walking speed and those who died during the surveillance the slowest while those who were admitted to an institution were in the middle. For cognitive capacity, poorest baseline scores were observed among those who were later institutionalized and highest for those who survived and were still community-dwelling at the end of follow-up.

The institutionalization rate was $24.4 / 1000$ person-years among those in the lowest third of walking speed and 10.1/1000 persons-years for those in the middle and highest thirds. For cognitive capacity principal score the corresponding figures were 21.0/1000 and 11.0/1000 person-years, respectively. To study the combined effects of mobility limitations and cognitive deficits, four exclusive study groups were formed. Institutionalization rate among those with both mobility limitations and cognitive deficits was 35.1/1000 persons-years, whereas the rate 
among those with solely mobility limitation was $15.6 / 1000$ and solely cognitive deficits 10.0/1000 person-years. The number of institutionalization cases and rates are presented in Figure 1.

Cox proportional hazards model was employed for estimating the relative risks of institutionalization. First, walking speed and cognitive capacity were entered into the model as independent variables as presented in Table 2. The crude risk for institutionalization was 2.55 (95\% confidence interval [CI] 1.45-4.49) higher for those in the lowest third of the dichotomized walking speed variable and 1.94 (95 \% CI 1.11-4.00) higher for those in the lowest third of cognitive capacity variable compared to those in the middle and highest thirds, respectively. A significant interaction between the variables $(\mathrm{p}<0.001)$ was observed. Secondly, the combined effects of mobility and cognitive capacity were tested. The unadjusted relative risk of institutionalization was 3.80 times greater (95 \% CI 1.85-7.77) among people with cooccurring mobility limitation and cognitive deficits compared to people with no limitations. Adjusting the model for education, living alone and comorbidity increased the risk to 4.89 (95\% CI 2.14-11.17). In this combined effects model, having solely mobility limitations or solely cognitive deficits increased the risk of institutionalization slightly but failed to reach statistical significance due to the small number of cases in the respective groups. 


\section{DISCUSSION}

Results of this study show that the presence of co-existing mobility limitations and cognitive deficits places older persons at a significantly higher risk for institutionalization compared to persons with no limitations or only a single limitation. The relative risk for institutionalization regarding combined mobility limitations and cognitive deficits was almost five-fold compared to those with no limitations.

Population-based studies have identified cognitive decline [2-4] and physical impairments [14] in non-demented populations as independent risk factors for institutionalization. However, previous research has not, to the best of our knowledge, addressed multiple co-existing limitations and impairments as predictors of institutionalization among older populations.

A sufficient mobility ability [22] and cognitive status [6] are prerequisites for independent community-dwelling. Moving about at home and its surroundings is essential for self-care and for maintaining a socially engaged lifestyle. Performing Instrumental Activities of Daily Living (IADLs) such as shopping and preparing food, and engaging in meaningful social interaction requires a sufficient cognitive capacity level. Moreover, physiologic [23] and cognitive reserves [24] help older persons withstand the deterioration of functional abilities which, upon depletion, might result in adverse health outcomes such as institutional care. In our study, it is most likely that persons who were classified at baseline into the highest and middle third in mobility and cognitive capacity had greater reserve capacity than those in the lowest thirds. Further, a person with decreased physical reserve capacity is more vulnerable to acute insults [23]. For instance, a person with substantial physiologic reserve would presumably be able to withstand the strain of for example an acute respiratory infection without losing functional independence or otherwise compensate for potential losses [25] with that reserve and thus avoid loss of independence. 
Similarly, it is probable that many of those in the lowest third of cognitive capacity had a substantially decreased cognitive reserve. Consequently, it is possible that they already suffered from mild cognitive impairment or preclinical dementia and thus had an increased risk of manifest dementia and subsequent dependence. Unfortunately, we do not know the immediate precipitating events of institutionalization for our participants and thus cannot confirm their underlying situation. In addition to individual resources, environmental and social elements such as availability of caregivers contribute to the need for institutional care. However, we adjusted our model with living alone and thus were able to control for social elements, at least to some extent.

In the study, co-occurrence of slow walking speed and cognitive deficits was higher than expected by chance only, Kappa coefficient $0.173, \mathrm{p}<0.001$. Furthermore, there is a growing body of evidence linking physical and cognitive decline [26-28], suggesting that they might share the same etiology or be causally related to each other so that one of the limitations precedes the other. A recent study found smoking and lower haemoglobin levels to be associated with combined physical and cognitive decline [28]. Decline in cognitive performance has been associated with decline in physical tasks such as walking at normal speed. Thus indicating that patterns of change in cognitive and physical performance are interrelated and that cognition plays a significant role in the execution of physical tasks [26]. On the other hand, inability to maintain a socially active lifestyle due to physical disability may accelerate cognitive decline [27]. In our study the causal nature of mobility limitations and cognitive decline cannot be determined.

The risk for institutionalization for persons with co-existing mobility limitations and cognitive decline increased in our study after adjusting for the comorbidity index. This may be related to the fact that problems inflicted by chronic diseases are often expressed through functional 
difficulties which, in turn, predict development of disability [29] and subsequent loss of independence.

This population-based prospective study had a long surveillance period. As studies often rely on self-reported data or estimations of institutional care [4], the use of local registers with exact dates on institutionalization is a definite advantage of our study. Another strong point of the study was the use of a performance-based measure, walking speed, for assessing physical function [15] instead of self-rated measures, which might yield biased information. In addition, psychometric tests indicating cognitive capacity were performed by trained psychologists.

In the study, we used distribution based cut-off values for walking speed and cognitive capacity, given that there is lack of 'golden standards' for cut-off points. We used maximal walking speed to measure mobility limitations [15]. Our age- and gender-specific cut-offs, $1.59-1.19 \mathrm{~m} / \mathrm{s}$, are similar to those who use maximal walking speed as an indicator of functional capacity [21] but obviously higher than for example shown in a recent study [30], which measured usual walking speed instead of maximal speed. In our study, cognitive capacity was measured with three psychometric tests [18-20]. At 5-year follow-up among 80- and 85-year-old people, in addition to the psychometric tests used here the Mini-Mental State Examination MMSE test [31], a widely used test for screening people at increased risk of dementia, was done. A significant correlation was found, $r=0.513, \mathrm{p}<0.001$. Further, an effort was made to test the validity of cutoffs for the principal component indicating cognitive capacity. The cognitive capacity principal component and MMSE scores were dichotomized into the lowest third vs. middle and highest thirds. The cut-off values on the MMSE were 25 and 24 for the 80 - and 85-year-olds, respectively [31]. The reliability for the dichotomized cognitive capacity principal component and MMSE was found to be moderate with a Kappa coefficient of $0.395, \mathrm{p}<0.001$. We thus 
conclude that a substantial proportion of people in the cognitive deficits category could have been defined as having mild cognitive impairment or preclinical dementia.

Our study included persons who were community-dwelling, did not have a diagnosis of dementia and were able to come to the research centre for an entire day and participate in multiple physical and psychometric tests. Twenty three percent of the target group did not come to the research centre for testing and consequently, it is more likely that persons with poor mobility and/or cognitive decline dropped out rather than persons with no limitations. We performed basic analysis on non-participants and found them to be older and have a higher comorbidity index than our participants, which lends support to our assumption. Consequently, the present study might have underestimated the risk of institutionalization at population level, which should be considered when interpreting our results. Even though our study was relatively small scale it nevertheless had a long surveillance period and provides new information on co-existing risk factors for institutionalization.

In conclusion, results show that co-existing decline in physical and cognitive status poses a significant risk for institutionalization. On the other hand, it should be noted that a decision for institutionalization is not just a result of the functional status and individual qualities but, to some extent, also a social decision reflecting current policies and available resources [4]. At the moment, interventions for reducing or postponing adverse outcomes such as institutionalization should target modifiable risk factors, such as physical limitations. Consequently, our results indicate that interventions targeted to improve even one limitation or prevent accumulation of these risk factors could significantly reduce the risk of institutionalization. 


\section{REFERENCES}

1. Tomiak M, Berthelot J-M, Guimond E, Mustard CA. Factors associated with nursinghome entry for elderly in Manitoba, Canada. J Gerontol A Biol Sci Med Sci 2000; 55A: M279-287.

2. Agüero-Torres H, von Strauss E, Viitanen M, Winblad B, Fratiglioni L. Institutionalization in the elderly: The role of chronic diseases and dementia. Crosssectional and longitudinal data from a population-based study. J Clin Epidemiol 2001; 54: 795-801.

3. Banaszak-Holl J, Fendrick AM, Foster NL, Herzog AR, Kabeto MU, Kent DM et al. Predicting nursing home admission. Estimates from a 7-year follow-up of nationally representative sample of older Americans. Alzheimer Dis Assoc Disord 2004; 18: 83-88.

4. Bharucha AJ, Pandav R, Shen C, Dodge HH, Ganguli M. Predictors of nursing facility admission: A 12-year epidemiological study in the United States. J Am Geriatr Soc 2004; 52: 434-439.

5. Laukkanen P, Leskinen E, Kauppinen M, Sakari-Rantala R, Heikkinen E. Health and functional capacity as predictors of community dwelling among elderly people. J Clin Epidemiol 2000; 53: 257-265.

6. Angel JL, Angel RJ, Aranda MP, Miles TP. Can families still cope? Social support and health as determinants of nursing home use in the older Mexican-origin population. $\mathbf{J}$ Aging Health 2004; 16: 338-354.

7. Jagger C, Spiers NA, Clarke M. Factors associated with decline in function, institutionalization and mortality of elderly people. Age Aging 1993; 22: 190-197.

8. Hays JC, Pieper CF, Purser JL. Competing risk of household expansion or institutionalization in late life. J Gerontol B Psychol Sci Soc Sci 2003; 58: S11-20. 
9. Boaz RF, Muller CF. Predicting the risk of "permanent" nursing home residence: The role of community help as indicated by family helpers and prior living arrangements. Health Serv Res 1994; 29: 391-414.

10. Tsuji I, Whalen S, Finucane TE. Predictors of nursing home placement in communitybased long-term care. J Am Geriatr Soc 1995; 43: 761-766.

11. Fransen M, Woodward M, Norton R, Robison E, Butler M, Campbell AJ. Excess mortality or institutionalization after hip fracture: Men are at greater risk than women. $\mathbf{J}$ Am Geriatr Soc 2002; 50: 685-690.

12. Verbrugge LM, Jette AM. The disablement process. Soc Sci Med 1994; 38: 1-14.

13. Miller EA, Weissert WG. Predicting elderly people's risk for nursing home placement, hospitalization, functional impairment, and mortality: A synthesis. Med Care Res Rev 2000; 57: 259-297.

14. Guralnik JM, Simonsick EM, Ferrucci L, Glynn RJ, Berkman LF, Blazer DG et al. A short physical performance battery assessing lower extremity function: Association with self-reported disability and prediction of mortality and nursing home admission. $\mathbf{J}$ Gerontol A Biol Sci Med Sci 1994; 49A: M85-94.

15. Guralnik JM, Ferrucci L, Pieper CF, Leveille SG, Markides KS, Ostir GV et al. Lower extremity function and subsequent disability: Consistency across studies, predictive models, and value of gait speed alone compared with a short physical performance battery. J Gerontol A Biol Sci Med Sci 2000; 55A: M221-231.

16. Heikkinen E. Background, design, and methods of the project. Scand J Soc Med Suppl 1997; 53: 1-18.

17. Aniasson A, Rundgren $\AA$, Sperling L. Evaluation of functional capacity in activities of daily living in 70-year-old men and women. Scand J Rehabil Med 1980; 12: 145-154.

18. Wechsler D. A standardized memory scale for clinical use. J Psychol 1945; 19: 87-95. 
19. Wechsler D. Manual for the Wechsler Adult Intelligence Scale (WAIS). New York: Psychological Corporation; 1955.

20. Schaie KW. Schaie-Thurstone Adult Mental Abilities Test, form OA. Palo Alto. CA: Consulting Psychologists Press; 1985.

21. Shinkai S, Watanabe S, Kumgai S, Fujiwara Y, Amano H, Yoshida H et al. Walking speed as a good predictor for the onset of functional dependence in a Japanese rural community population. Age Ageing 2000; 29: 441-446.

22. Judge JO, Schechtman K, Cress E, the FICSIT Group. The relation between physical performance measures and independence in instrumental activities of daily living. J Am Geriatr Soc 1996; 44: 1332-1341.

23. Fried LP, Ferrucci L, Darer J, Williamson JD, Anderson G. Untangling the concepts of disability, frailty, and comorbidity: Implications for improved targeting and care. $\mathbf{J}$ Gerontol A Biol Sci Med Sci 2004; 59A: M255-263.

24. Richards M, Deary IJ. A life course approach to cognitive reserve. A model for cognitive aging and development. Ann Neurol 2005; 58: 617-622.

25. Tinetti ME, Inouye SK, Gill TM, Doucette JT. Shared risk factors for falls, incontinence, and functional dependence. JAMA 1995; 273: 1348-1353.

26. Tabbarah M, Crimmins EM, Seeman TE. The relationship between cognitive and physical performance: MacArthur studies of successful aging. J Gerontol A Biol Sci Med Sci 2002; 57A: M228-235.

27. Bassuk SS, Glass TA, Berkman LF. Social disengagement and incident cognitive decline in community-dwelling elderly persons. Ann Intern Med 1999; 131: 165-173. 
28. Atkinson HH, Cesari M, Kritchevsky SB, Penninx B, Fried LP, Guralnik JM et al. Predictors of combined cognitive and physical decline. J Am Geriatr Soc 2005; 53: 1197 1202.

29. Fried LP, Guralnik JM. Disability in older adults: Evidence regarding significance, etiology, and risk. J Am Geriatr Soc 1997; 45: 92-100.

30. Cesari M, Kritchevsky SB, Penninx B, Nicklas BJ, Simonsick EM, Newman AB, et al. Prognostic value of usual gait speed in well-functioning older people- Results from the Health, Aging and Body Composition Study. J Am Geriatr Soc 2005; 53: 1675-1680.

31. Folstein MF, Folstein SE, McHugh PR. "Mini-mental state.” A practical method for grading the cognitive status of patients for the clinician. J Psychiatr Res 1975; 12: 189198.

\section{Acknowledgements}

We are grateful to Ritva Sakari-Rantala for gathering the data on long-term institutionalization. We also like to thank Pertti Era for his comments on the manuscript. 
Table 1. Baseline characteristics of those who at the end of the follow-up were community-dwelling, institutionalized and those who died during the surveillance without first being institutionalized 


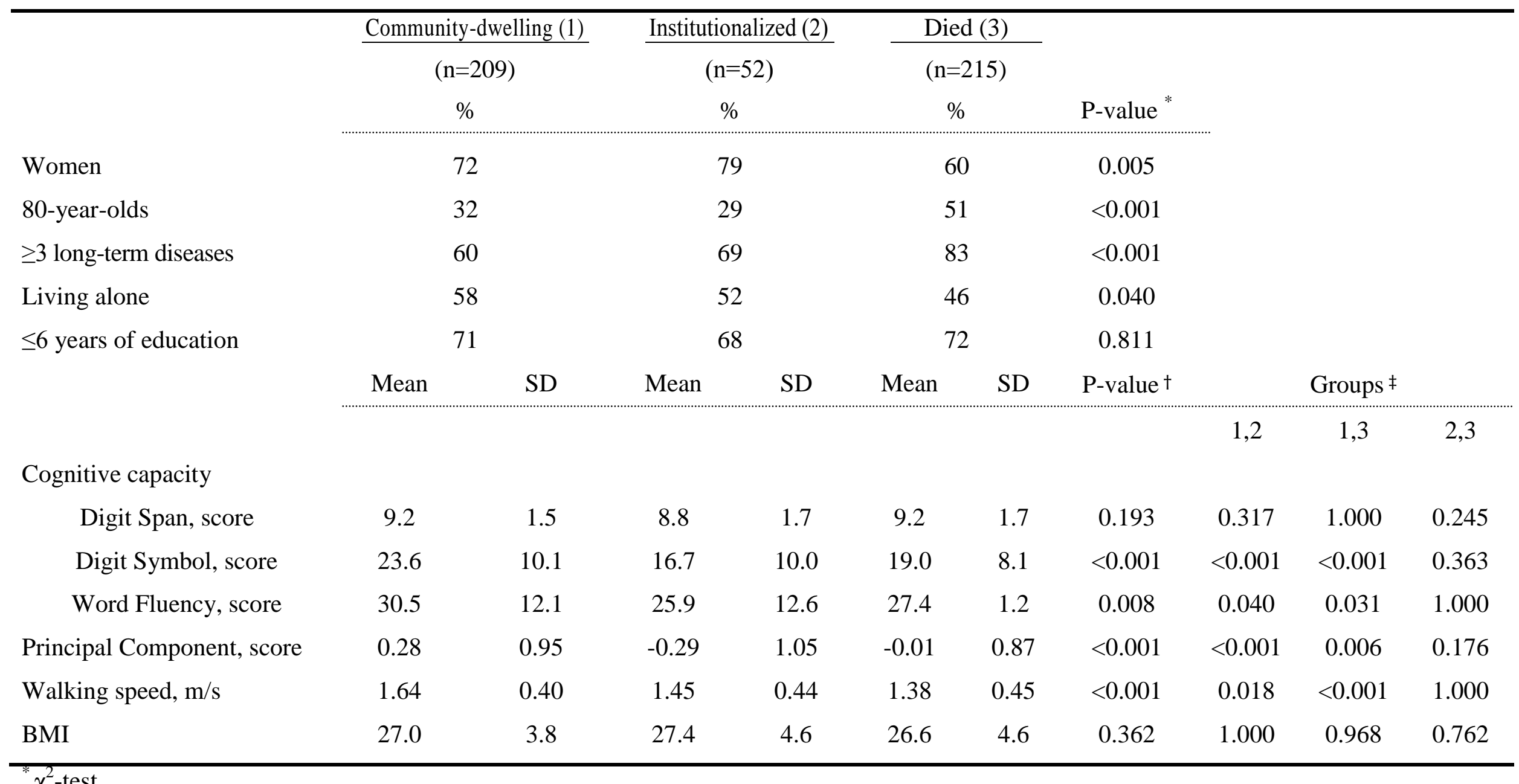

${ }^{*} \chi^{2}$-test.

${ }^{\dagger}$ Based on one-way ANOVA F-test score.

‡Paired comparisons with Bonferroni’s test for homogenous and Tamhane’s test for non-homogenous variances. 
Table 2. Relative risks (RR) and $95 \%$ confidence intervals (CI) of long-term care according to baseline mobility and cognitive capacity 


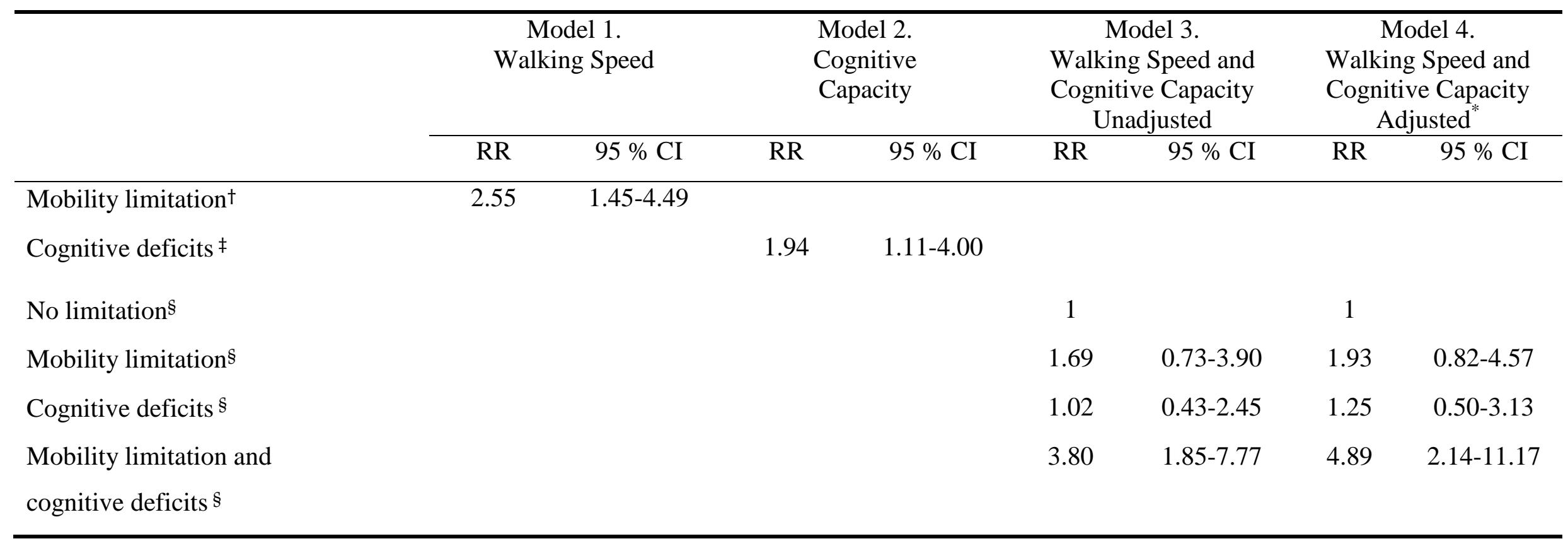

* Adjusted for education, living alone and co-morbidity.

†Lowest third of walking speed vs. middle and highest third.

‡Lowest third of cognitive capacity principal component score vs. middle and highest third.

$\S$ Groups formed based on walking speed and cognitive capacity tests dichotomized according to distribution based cut-offs at the lowest third vs. middle and highest third. 


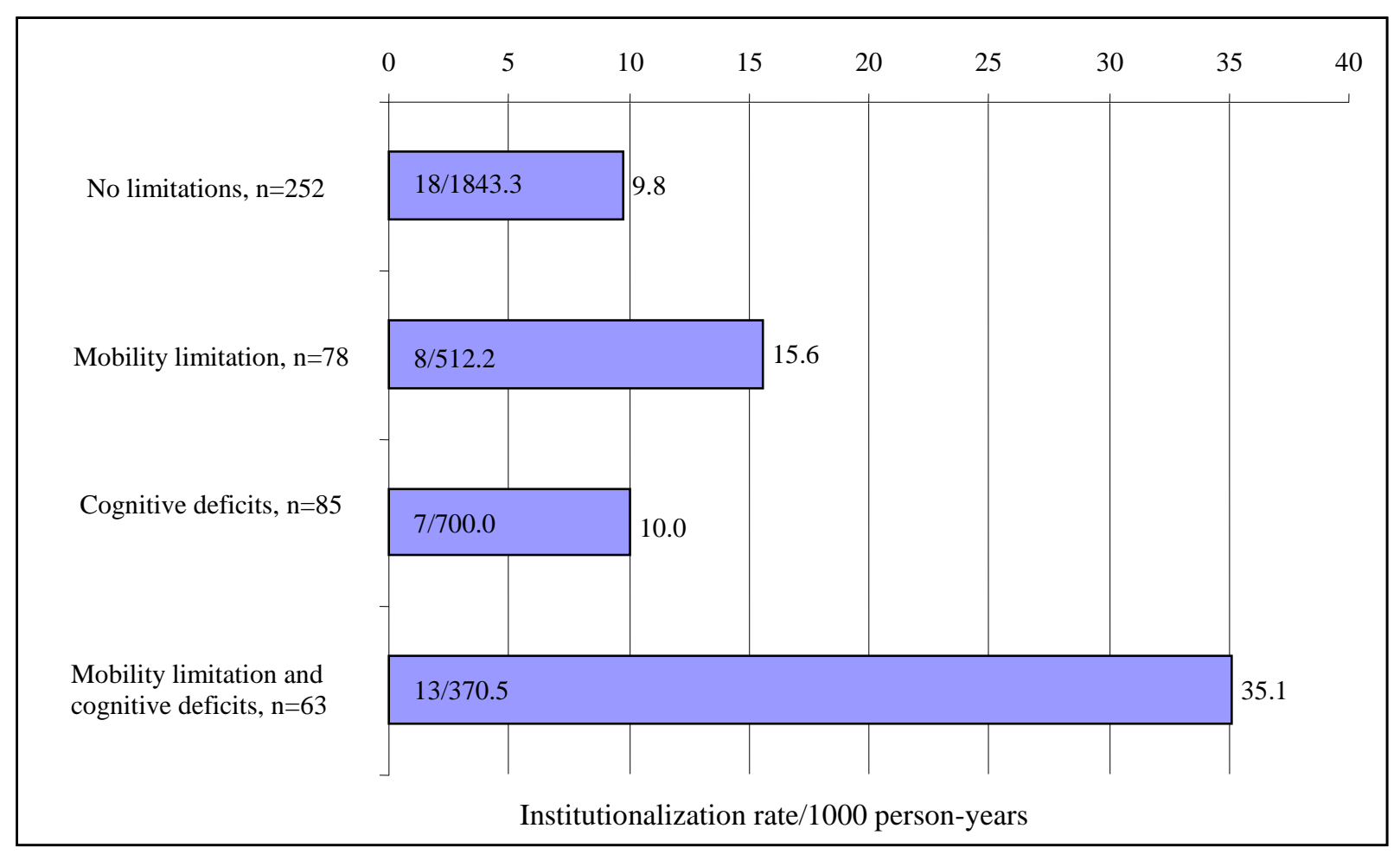


Figure 1. Institutionalization rates per 1000 person-years, long-term care cases and person-years according to study groups during the 10-year surveillance period. Groups were formed based on walking speed and cognitive capacity test dichotomized according to distribution based cutoffs at the lowest third vs. middle and highest third 